\title{
Modulation of Bone's Sensitivity to Low-Intensity Vibrations by Acceleration Magnitude, Vibration Duration, and Number of Bouts
}

\author{
Stefan Judex, Timothy Koh*, and Liqin Xie \\ Department of Biomedical Engineering \\ Stony Brook University \\ Stony Brook, NY 11794, USA \\ *Department of Kinesiology \& Nutrition \\ University of Illinois at Chicago \\ Chicago, IL 60612, USA
}

$\begin{array}{ll}\text { Running Title: } & \text { Sensitivity of Bone to Vibration Variables } \\ \text { Submitted to: } & \text { Osteoporosis International }\end{array}$

Disclosure: Stefan Judex and Timothy Koh own (provisional) patents regarding the application of vibrations to the musculoskeletal system. Liqin Xie has no conflict of interest.

\section{Correspondence to:}

Stefan Judex, Ph.D.

Department of Biomedical Engineering

Bioengineering Building, Rm 213

Stony Brook University

Stony Brook, NY 11794-5281

Phone: 631-632-1549

FAX: 631-632-8577

e-mail: stefan.judex@stonybrook.edu 


\section{SUMMARY}

Variables defining vibration based biomechanical treatments were tested by their ability to affect the musculo-skeleton in the growing mouse. Duration of a vibration bout, but not variations in vibration intensity or number of vibration bouts per day, was identified as modulator of trabecular bone formation rates.

INTRODUCTION

Low-intensity vibrations (LIV) may enhance musculoskeletal properties but little is known regarding the role that individual LIV variables play. We determined whether acceleration magnitude and/or the number and duration of daily loading bouts may modulate LIV efficacy.

\section{MetHODS}

LIV was applied for $3 w k$ to $8 w k$ old mice at either $0.3 \mathrm{~g}$ or $0.6 \mathrm{~g}$, the number of daily bouts was one, two, or four, and the duration of a single bout was 15,30 , or $60 \mathrm{~min}$. A frequency of $45 \mathrm{~Hz}$ was used throughout.

\section{RESULTS}

LIV induced tibial cortical surface strains in 4 mo old mice of approximately $10 \mu \varepsilon$ at $0.3 \mathrm{~g}$ and $30 \mu \varepsilon$ at $0.6 \mathrm{~g}$. In trabecular bone of the proximal tibial metaphysis, all single daily bout signal combinations with the exception of a single $15 \mathrm{~min}$ daily bout at $0.3 \mathrm{~g}$ (i.e., single bouts of $30 \mathrm{~min}$ and $60 \mathrm{~min}$ at $0.3 \mathrm{~g}$ and $15 \mathrm{~min}$ and $30 \mathrm{~min}$ at $0.6 \mathrm{~g}$ ) produced greater bone formation rates (BFR/BS) than in controls. Across all signal combinations, $30 \mathrm{~min}$ and $60 \mathrm{~min}$ bouts were significantly more effective than $15 \mathrm{~min}$ bouts in raising BFR/BS above control levels. Increasing the number of daily bouts or partitioning a single daily bout into several shorter bouts did not potentiate efficacy and 
in some instances led to BFR/BS that was not significantly different from those in controls. Bone chemical and muscle properties were similar across all groups.

\section{CONCLUSIONS}

These data may provide a basis towards optimization of LIV efficacy and indicate that in the growing mouse skeleton, increasing bout duration from $15 \mathrm{~min}$ to $30 \mathrm{~min}$ or $60 \mathrm{~min}$ positively influences BFR/BS.

\section{KEYWORDS}

Low intensity vibrations; bone formation; bone resorption, trabecular bone; cortical bone; muscle mass; mechanical strain 


\section{Introduction}

High levels of physical activity can confer substantial benefits to the growing and young adult skeleton, aiding in the accretion of tissue and attenuating the erosion of surfaces, ultimately reducing fracture rates [1-4]. However, strenuous activities should not necessarily be prescribed to young untrained individuals as fracture rates in children may be directly related to physical activities [5]. As an alternative to high loads associated with exercise, the application of lowintensity vibrations (LIV) to the musculoskeleton as a means of raising bone mass without exercising per se has been suggested. In animal models, exposure of the developing musculoskeleton to low intensity ( $<1 \mathrm{~g}$ with $\boldsymbol{g}$ referring to Earth's gravitational acceleration) vibrations enhanced bone formation and bone strength, attenuated resorption, and augmented muscle cross-sectional area [6-8].

No large-cohort randomized prospective study has been completed but smaller clinical studies have been largely consistent with results from animal models. For instance, LIV was able to raise bone mass in young adult women and men as well as in postmenopausal women [9-11], disabled children $[12,13]$, or prevent bone loss in post-menopausal women [14]. While LIV exposure has been primarily associated with a positive skeletal response, several investigations did not detect treatment effects in animal [15] or clinical studies [16]. Further, comparisons between the magnitudes of the treatment effect reveal a large range [12, 14]. Presumably, at least some differences among study outcomes are linked to variables including age, species, genetics, gender, experimental duration, or experimental techniques while others may reflect specific differences in the applied mechanical signal. 
The waveform used for vibrations is typically sinusoidal in nature and can be specified by the frequency (number of oscillations per second, $\mathrm{Hz}$ ) and the magnitude of the induced peak acceleration (expressed typically as multiples of $\boldsymbol{g}$ as defined above). In both animal and clinical vibration studies, the most commonly utilized frequencies range from $15 \mathrm{~Hz}$ to $90 \mathrm{~Hz}$. Variability in the applied peak acceleration has even been greater with differences larger than two orders of magnitude (from $0.1 \mathrm{~g}$ to about $30 \mathrm{~g}$ ). Other variables defining a loading protocol include the duration of a loading session [6, 9] and the number of loading sessions per day [17].

Manipulation of these variables may augment LIV efficacy but little is known about their individual roles in orchestrating the biologic response and even less is known about interactions between vibration variables. In contrast to bone adaptation experiments using lower-frequency mechanical signals $(<10 \mathrm{~Hz})$, mechanotransduction of vibrations is not tuned to the magnitude of the induced matrix deformation or one of its byproducts like fluid shear $[18,19]$. For instance, exposure to $0.3 \mathrm{~g}$ vibrations can be less effective than $0.1 \mathrm{~g}$ vibrations in increasing trabecular bone volume in a murine model [20]. Different vibration frequencies have rarely been directly compared to each other and at least in the rodent musculoskeleton may have a preference for $90 \mathrm{~Hz}$ over $45 \mathrm{~Hz}$ vibrations [21-23].

Because of the large number of vibration variables including their potential interactions that may play a role in modulating bone's response, an exhaustive in vivo optimization study using a full factorial design is impractical. Here, in an effort towards identifying variables that can enhance the response of the growing skeleton to LIV, we hypothesized that increasing the number of daily vibration bouts [17] and partitioning a single daily bout into multiple discrete 
bouts interspersed with rest [24], but not bout duration [9] or vibration magnitude [20], will modulate the LIV induced increase in bone formation rates.

\section{Methods}

\section{Experimental Design and Mice}

Experimental procedures were approved by the Institutional Animal Care and Use Committee (IACUC) at Stony Brook University. One hundred and thirty 6-week-old male BALB/cByJ (BALB) mice were obtained from The Jackson Laboratory (Bar Harbor, ME). At 8wk of age, mice were randomly divided into 12 groups comprising nine experimental groups, one baseline control group and two age-matched (sham) control groups. At $8 \mathrm{wk}$ of age, mice are reproductively mature but bone elongation is still occurring and cortical bone in particular is still expanding [7]. The signal frequency of the vertical whole body vibration that each of the nine experimental groups was exposed to was identical $(45 \mathrm{~Hz})$ while the peak acceleration magnitude of the LIV signal (either $0.3 \mathrm{~g}$ or $0.6 \mathrm{~g}$ ), the number of bouts per day (one, two, or four), and the duration of each bout (15min, $30 \mathrm{~min}$, or $60 \mathrm{~min}$ ) was varied. The levels of each LIV variable (\# bouts, bout duration, g) were selected within a relatively narrow range that reflected use of these variables in previous clinical and animal studies, practicality, and potential safety issues. Experimental duration was $3 w k$ for all groups except baseline controls $(B C, n=5)$ which were sacrificed at $8 w k$ of age. Sample size for BC mice was relatively small because its purpose was to provide basic information on trabecular, cortical, and muscle variables at baseline and was not intended for direct comparisons with experimental groups. It was confirmed that average body mass was similar between all groups at start of the study. 
The nine experimental groups comprised: (1-3) $0.3 \mathrm{~g}, 15 \mathrm{~min} /$ bout for either 1 bout/d $(0.3 \times 15 \times 1, n=11), 2$ bouts/day $(0.3 \times 15 \times 2, n=11)$, or 4 bouts/day, $(0.3 \times 15 \times 4, n=12),(4-5) 0.3 g$, $30 \mathrm{~min} /$ bout, for either 1 bout/d $(0.3 \times 30 \times 1, \mathrm{n}=12)$ or 2 bouts/day $(0.3 \times 30 \times 2, \mathrm{n}=11),(6) 0.3 \mathrm{~g}$,

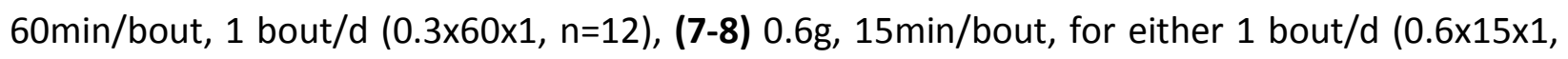
$\mathrm{n}=11)$ or 2 bouts/day $(0.6 \times 15 \times 2, n=11)$, and (9) $0.6 \mathrm{~g}, 30 \mathrm{~min} /$ bout, 1 bout $/ \mathrm{d}(0.6 \times 30 \times 1, \mathrm{n}=11)$ (Table 1). For regimes with 2 bouts/d, bouts were separated by $6 h$; 4 bouts/d were separated by 3h each. All regimes were applied 5d/wk with two consecutive rest days. Age-matched control mice were handled similarly to experimental mice and exposed to an inactive vibrating plate either once a day for $15 \min (0 \times 15 \times 1, n=12)$ or twice a day for $30 \min (0 \times 30 \times 2, n=12)$ (Table 1). All mice in a given group received LIV (or sham LIV for controls) at the same time with up to four plastic containers placed on the vertically oscillating plate, each container housing up to five mice $(\mathrm{LxWXH}, 35 \times 24 \times 16 \mathrm{~cm})$. During treatment, mice were allowed to freely roam the container.

When not engaged in the mechanical intervention, all mice were single-housed in regular mouse cages $\left(22^{\circ} \mathrm{C}\right)$ and exposed to $12 \mathrm{~h}$ light/dark cycles with ad libitum access to water and standard rodent chow (LabDiet RMH 3000, LabDiet, St. Louis, MO). To enable measurement of dynamic indices of bone formation, mice were injected (i.p., $0.5 \mathrm{ml})$ with calcein $(15 \mathrm{mg} / \mathrm{kg}$ ) dissolved in phosphate buffered saline on days 15 and 20 of the experimental protocol with the last injection $48 \mathrm{~h}$ prior to sacrifice. Mice were euthanized by carbon dioxide inhalation.

\section{Tissue Preparation}

After the $3 w k$ experimental duration, the right tibia was harvested and submerged in $70 \%$ ethanol for micro-computed tomography $(\mu \mathrm{CT})$ and histomorphometry. Length of the right tibia 
was measured with digital calipers. The left tibia was transected at the proximal and distal diaphysis (Buehler Isomet Slow Speed Saw) and fixed overnight in 10\% neutral buffered formalin for staining of osteoclastic resorption via tartrate resistant acid phosphatase (TRAP) [6]. The right soleus was harvested and pinned to a wooden rod in an attempt to retain the original in vivo muscle length. The pinned muscle specimens were embedded in TBS tissue freezing medium (Triangle Biomedical Sciences, Durham, NC), snap frozen in cooled isopentane with liquid nitrogen, and stored in an isopentane-filled Eppendorf tube at $-80^{\circ} \mathrm{C}$ until sectioning [25]. All analyses described below were performed by a single operator who was blinded to the identity of the samples.

\section{Bone Strain Measurements}

To estimate differences in the strain environment of the tibia between the $0.3 \mathrm{~g}$ and $0.6 \mathrm{~g}$ mechanical signal, cortical surface bone strains generated in the proximal tibia were measured in two additional BALB mice. Because of the small size of the mouse tibia at 8wk of age, young adult (4mo old) mice were used. It is entirely possible that the strains recorded in these older mice do not reflect the absolute values of peak strains induced in 8-10wk old mice but the main purpose of strain gaging these mice was to get an approximate estimate for the relative change in strain magnitude when the magnitude of the LIV acceleration is raised from $0.3 \mathrm{~g}$ to $0.6 \mathrm{~g}$ (and to relate this change in strain magnitude to the difference in bone formation between mice subjected to $0.3 \mathrm{~g}$ and $0.6 \mathrm{~g}$ LIV signals). Under isoflurane anesthesia, a miniature single-element

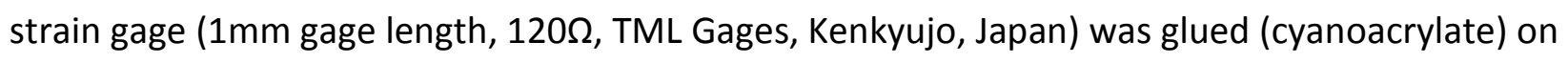
the antero-medial surface of the proximal tibia [6]. Upon recovery from surgery (1-2h), and with 
the animal standing on the vibrating plate, strain data were collected over two $10 \mathrm{sec}$ trials. Strain gage signals were amplified (SX500, Syminex Inc, Mt. Arlington, NJ) with an excitation of $4 \mathrm{~V}$ and a $1000 x$ gain, and acquired at a sampling rate of $1000 \mathrm{~Hz}$. This setup collected in vivo strain data at a resolution of approximately 0.5 microstrain $[6,26]$. Strain data were plotted and the amplitude of the oscillatory strain reading was determined.

\section{Microcomputed Tomography}

We scanned $5 \mathrm{~mm}$ of the right proximal tibia of all mice at a voxel size of $6 \mu \mathrm{m}(55 \mathrm{kV}, 145 \mathrm{~mA}$, 250ms integration time, 1000 projections) on a $\mu$ CT 40 (Scanco Medical, SUI). The diaphysis was scanned at $12 \mu \mathrm{m}$ voxel size using the same scan parameters. The trabecular VOI in the proximal metaphysis was $600 \mu \mathrm{m}$ in length starting $500 \mu \mathrm{m}$ distal of the $\mu \mathrm{CT}$ slice in which primary spongiosa (non-resolvable as separate struts) separates at the center of a tibial cross-section (most distal point of the growth plate). Trabecular bone was manually separated from cortical bone with contour lines drawn about $50 \mu \mathrm{m}$ from the endocortical surface, marking the outside boundary of the VOI. Cortical bone was analyzed from the metaphysis (surrounding the trabecular VOI) as well as the middiaphysis (defined at 50\% of tibial length comprising 10 slices). Global thresholds were determined, one each for trabecular and cortical bone, by visual comparisons between segmented and raw grey-scale images from at least two mice per group until the segmented images matched the morphology of the grey-scale images.

For trabecular regions, bone volume fraction (BV/TV), connectivity density (Conn.D), trabecular thickness (Tb.Th), and trabecular number (Tb.N) were determined. For cortical bone, total area (Tt.Ar), bone area (Ct.Ar), and bone marrow area (Ma.Ar) were calculated. 


\section{Histomorphometry}

Following tomographic scanning, the right proximal and diaphyseal tibia were cut (Buehler Isomet Slow Speed Saw, Lake Bluff, IL) and embedded in methyl methacrylate resin (MMA) using a standard protocol [6]. The proximal specimens were sectioned longitudinally in the center to yield $5 \mu \mathrm{m}$ frontal sections (RM 2165 Microtome, Leica, Bensheim, Germany) while diaphyseal samples were sectioned at midpoint (40 $\mu$ m thick) with a diamond wire saw (Well Diamond Wire Saws, Norcross, GA). The evaluated regions in the metaphysis and diaphysis were similar to the regions scanned by $\mu \mathrm{CT}$. The trabecular ROI spanned $800 \mu \mathrm{m}$ in the secondary spongiosa of the metaphysis starting $400 \mu \mathrm{m}$ distal from the border of the growth plate. Because of a lack of consistent double labels at the endocortical surface of the middiaphysis and the periosteal surface of the metaphysis, these surfaces were excluded from the analysis. Bone formation rates (BFR/BS, $\mu \mathrm{m}^{3} / \mu \mathrm{m}^{2} / \mathrm{yr}$ ) were calculated as the product of mineral apposition rate (MAR, $\mu \mathrm{m} /$ day) and mineralizing surface (MS/BS, $\%$, calculated as the sum of percent double label and $1 / 2 \mathrm{x}$ percent single label) by histomorphometric software (Osteomeasure, OsteoMetrics Inc., Atlanta, GA). Standard nomenclature was adopted [27].

\section{TRAP Staining}

Tartrate resistant acid phosphatase (TRAP), an indicator of osteoclastic resorptive by-products, was used to estimate trabecular bone resorption in situ [28]. The left proximal tibia was fixed fresh in $10 \%$ neutral buffered formalin overnight and decalcified in $2.5 \%$ formic acid $(\mathrm{pH} 4.2)$ for 4d. Upon dehydration, samples were embedded in glycol methacrylate (GMA) according to the 
manual of the JB-4 embedding kit (Polysciences, Warrington, PA). Frontal sections were cut (7 $\mu \mathrm{m})$ and stained for TRAP activity. Hexazotization was achieved by mixing equal amounts of $4 \% \mathrm{NaNO}_{2}$ and $4 \%$ pararosaniline solutions. Naphthol-ASTR-phosphate (Sigma, St. Louis, MO) was used as a substrate and the enzyme reaction was carried out in the presence of tartrate $(10 \mathrm{mM})$ to demonstrate TRAP activity ( $\mathrm{pH} 5$ in $0.1 \mathrm{M}$ acetate buffer). Sections were counter-stained with methyl green to improve contrast. The ratio of trabecular osteoclast surface (Oc.S) to bone surface (BS) was determined (Osteomeasure) in a region that matched the histomorphometric analysis.

\section{Chemical Composition}

A critical requirement of any effective biochemical or mechanical intervention is that the bone which is formed as a result of the treatment is of high quality. To test whether LIV-induced bone formation was chemically different from bone forming in control mice, synchrotron infrared microspectroscopy was performed on newly formed bone enclosed by the double calcein labels as described previously [29]. To this end, chemical measurements were taken from five $12 \times 12$ $\mu \mathrm{m}$ spectra within two randomly selected cortical and trabecular regions each. Ratios of phosphate-to-protein, carbonate-to-protein, carbonate-to-phosphate, collagen cross-linking, acid phosphate-to-total phosphate, and crystallinity were compared between age-matched controls ( $n=8$ randomly selected) and the $0.3 \times 15 \times 2$ group ( $n=7$ randomly selected), as a representative LIV group in which bone formation rates were significantly up-regulated by vibrations. 


\section{Muscle Histology}

Prior to sectioning, each soleus was re-embedded and snap frozen in Tissue-Tek OCT [25]. Multiple frozen transverse sections were cut $(8 \mu \mathrm{m})$ from the mid-belly region with a cryostat at $-20^{\circ} \mathrm{C}$ (CM3050S microtome, Leica, Bensheim, Germany). To evaluate potential changes in myofibers, myocyte myosin ATPase activity was stained histochemically by previously verified methods to classify slow- and fast-twitch fibers. Pre-incubation $(\mathrm{pH} 10.4)$ inactivated the myosinATPase enzyme in type I fibers (slow) and the remaining active ATPase enzyme in type II fibers (fast) produced a black insoluble compound. Cross-sectional images of the soleus were taken under a light microscope with a 4 X objective (Axiovert 200M, Zeiss, Germany). Muscle crosssectional areas and numbers of type I, type II, and total muscle fibers were evaluated with Image J (NIH, Bethesda, MD).

\section{Statistical Analysis}

All data were expressed as mean \pm SD. A total of 23 variables were tested but the principal outcome variables were trabecular BFR/BS and total muscle cross-sectional area. First, an unpaired t-test showed that there were no significant differences between the two sham-LIV groups, $0 \times 15 \times 1$ and $0 \times 30 \times 2$, for any outcome variable and these two groups were pooled into a single age-matched control group $(A C)$ to which LIV groups were compared to. Second, the $B C$ and $\mathrm{AC}$ groups were compared with an unpaired t-test to determine normal growth related changes in outcome measures occurring during the 3 wk period. Third, three separate one-way ANOVAs were performed. The first ANOVA involved ten groups, the AC group and the nine LIV groups. When a one way ANOVA for a variable was significant, a Student-Newman-Keuls (SNK) 
post-hoc test assessed differences between AC and any given LIV group. When a one way ANOVA for a variable was significant, two additional one-way ANOVA were performed to test for significant differences among control and $0.3 \mathrm{~g}$ LIV groups (AC, $0.3 \times 15 \times 1,0.3 \times 15 \times 2,0.3 \times 15 \times 4$, $0.3 \times 30 \times 1,0.3 \times 30 \times 2,0.3 \times 60 \times 1)$ and among control and $0.6 \mathrm{~g}$ LIV groups (AC, $0.6 \times 15 \times 1,0.6 \times 15 \times 2$, $0.6 \times 30 \times 1)$. Finally, three-way ANOVA was used to test whether acceleration magnitude, bout duration, and number of bouts had main effects and interactions on BFR/BS, the principal outcome variable for bone in this study.

In exploratory studies like this that seek to generate new hypotheses, it is typical not to adjust for Type I errors that might occur due to use of multiple outcome variables [30]. Further, while we present a large number of outcome variables, we stress that the principal outcome variables relied upon were trabecular BFR/BS for bone and total cross-sectional area for muscle. Statistical significance was set at 0.05 throughout (SPSS 22.0, IBM, New York, NY).

\section{Results}

Strain Magnitudes at $0.3 \mathrm{~g}$ versus $0.6 \mathrm{~g}$

The accelerometer attached to the vibration plate confirmed the sinusoidal LIV pattern of the vertically oscillating vibration plate (Fig. 1A). Concurrent in vivo recordings from a strain gage attached to the cortical tibial metaphysis demonstrated transmissibility of the mechanical signal into the tibia as indicated by sinusoidal strain patterns of the same frequency (Fig. 1B). Standing on an inactive plate induced a quasi-static compressive strain of approximately $70 \mu \varepsilon$ in magnitude (Fig. 1B). Superimposed upon the strain level associated with weightbearing, LIV applied at a frequency of $45 \mathrm{~Hz}$ and $0.3 \mathrm{~g}$ induced dynamic peak bone strains of approximately 
$10 \mu \varepsilon$ at the antero-medial surface of the proximal tibia (Fig. 1B). Doubling the peak acceleration to $0.6 \mathrm{~g}$ raised dynamic peak strain magnitudes to approximately $30 \mu \varepsilon$ (Fig. 1B).

Animals and Changes in Outcome measures during the 3wk Period in Control Mice

One mouse in the $0.3 \times 15 \times 2$ group died of reasons unrelated to the protocol, reducing this group from $n=11$ to $n=10$. Changes occurring through normal development in the growing male BALB mouse were inferred by comparing 8 wk-old baseline controls to 11 wk-old age-matched controls. During the $3 w k$ duration of the protocol, body mass increased by $19 \%(p<0.05)$ and the tibia extended its length by $5 \%(p<0.05)$ (Table 2$)$. In the trabecular metaphysis, BFR/BS decreased by $57 \%$, associated with a $37 \%$ drop in MAR and a $29 \%$ decrease in MS/BS (all $p<0.05$, Table 2 ). The prevalence of osteoclastic activity was not different between BC and AC mice (Table 2). Trabecular bone volume fraction (BV/TV) was $27 \%$ greater $(p<0.05)$ at the end of the experimental period (Fig. 2), caused primarily by $17 \%$ thicker trabeculae $(p<0.05$, Table 2 ). At the endocortical surface of the metaphysis, BFR/BS declined by $50 \%(p<0.05$, Fig. 3), resulting from a $42 \%(p<0.05)$ decrease in mineral apposition rate and a $13 \%(p<0.05)$ decrease in mineralizing surfaces (data not shown). At the periosteal surface of the middiaphysis, similar reductions in BFR/BS (46\%, p<0.05, Fig. 3) and MAR (31\%, p<0.05, data not shown) but not MS/BS (data not shown), were observed. While growth did not significantly affect cortical $\mu \mathrm{CT}$ outcome variables in the metaphysis, cortical bone area increased by $5 \%$ in the middiaphysis $(p<0.05$, Table 5$)$. There were also no differences in any histologic index of the soleus muscle between baseline and age-matched controls (Table 4). 


\section{Body Mass, Tibial Length, Chemical Properties, Muscle}

There was no significant difference in body mass and tibial length between groups at $11 \mathrm{wk}$ of age

(Table 2). There were also no differences in bone chemical properties when comparing newly

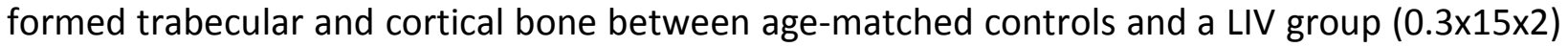
in which the intervention significantly increased BFR/BS (Table 3). No differences in muscle histological properties, including fiber diameter, number, and type were detected between agematched and experimental groups (Table 4).

\section{Bone Formation (trabecular)}

Every single mouse showed double labels in trabecular bone of the metaphysis. Using a threeway ANOVA on trabecular BFR/BS (Table 2), the principal outcome variable, bout duration but not acceleration magnitude or number of bouts had a significant main effect across all LIV groups with mice subject to $15 \mathrm{~min}$-bouts having lower trabecular BFR/BS than mice treated with $30 \mathrm{~min}$ or $60 \mathrm{~min}$ bouts. There was no difference between $30 \mathrm{~min}$ and $60 \mathrm{~min}$ bouts. There were also no significant interactions among the three factors. Trabecular BFR/BS results below were organized by hypotheses regarding the effects of individual LIV variables.

\section{Bout duration}

We first examined LIV duration for single bouts at $0.3 \mathrm{~g}$ and $0.6 \mathrm{~g}$. At a LIV amplitude of $0.3 \mathrm{~g}$ applied for $15 \mathrm{~min}$ once a day $(0.3 \times 15 \times 1)$, no difference in BFR/BS was detected when compared to the normal control group. However, increasing the duration of the bout to $30 \mathrm{~min}$ at the same amplitude $(0.3 \times 30 \times 1)$ led to $83 \%(p<0.05)$ greater BFR compared to controls (Fig. 4A, Table 2$)$. 
Extending the duration further to $60 \mathrm{~min}(0.3 \times 60 \times 1)$ provided a similar benefit $(88 \%, \mathrm{p}<0.05)$ for BFR/BS to that provided by $30 \mathrm{~min}$ (Fig. 4A, Table 2). At an acceleration amplitude of $0.6 \mathrm{~g}$ and a bout duration of $15 \mathrm{~min}(0.6 \times 15 \times 1), \mathrm{BFR} / \mathrm{BS}$ was $58 \%(\mathrm{p}<0.05)$ greater than in controls (Fig. 4A, Table 2). Extending the duration to $30 \mathrm{~min}$ per bout $(0.6 \times 30 \times 1)$ yielded an $84 \%(p<0.05)$ difference to AC (Fig. 4A, Table 2). Differences between individual LIV regimes were not significant.

Number of daily bouts

We then considered whether increasing the number of vibration bouts per day exerts a positive effect on BFR/BS in trabecular bone of the metaphysis at $0.3 \mathrm{~g}$ and $0.6 \mathrm{~g}$. Using $15 \mathrm{~min}$ per bout at 0.3g, a signal that did not raise BFR/BS when applied as a single bout per day (Fig. 4A, Table 2), doubling bout number to two bouts/d $(0.3 \times 15 \times 2)$ produced $62 \%(p<0.05)$ greater BFR/BS compared to AC (Fig. 4B, Table 2). Further increasing the number of bouts to four per day (0.3gx15minx4) yielded BFR/BS that were not significantly different from AC (Fig. 4B, Table 2). A similar nonlinearity was observed for $0.6 \mathrm{~g}$ vibrations applied for $15 \mathrm{~min}$; while one bout per day of this signal significantly raised BFR/BS over control levels, two bouts rendered this signal ineffective (Fig. 4B, Table 2 ). For the $30 \mathrm{~min}$ per bout $0.3 \mathrm{~g}$ signal, both one and two daily $30 \mathrm{~min}$ bouts produced BFR/BS that were $85 \%$ greater $(p<0.05)$ than in controls (Fig. 4B, Table 2). No significant differences between individual LIV groups were detected.

\section{Partitioning a single daily bout into multiple daily bouts}

Lastly, we investigated the effect of splitting a given LIV duration delivered as single bout into several bouts per day. At $0.3 \mathrm{~g}$, a daily vibration duration of 60 min caused increased BFR/BS when 
provided as a single bout (Fig. 4A, Table 2). Dividing this one bout of $60 \mathrm{~min}$ into two daily bouts of 30min LIV $(0.3 \times 30 \times 2)$ produced BFR/BS that were significantly different from that of controls (Fig. 4C, Table 2). Further dividing the $60 \mathrm{~min}$ vibration bout into four daily bouts of $15 \mathrm{~min}$ $(0.3 \times 15 \times 4)$ produced BFR/BS that were not significantly different from controls (Fig. 4C, Table 2 ). The one-bout vibration regimes of $0.3 \mathrm{~g}$ applied for $30 \mathrm{~min}$ and $0.6 \mathrm{~g}$ applied for $30 \mathrm{~min}$ had similarly elevated BFR/BS as the $0.3 \mathrm{~g} 60 \mathrm{~min}$ regime described above. Partitioning the $30 \mathrm{~min} 0.3 \mathrm{~g}$ and $0.6 \mathrm{~g}$ regimes into two discrete bouts of $15 \mathrm{~min}$ yielded BFR/BS for $0.3 \times 15 \times 2$ that was significantly greater than in AC, while BFR/BS for $0.6 \times 15 \times 2$ was indistinguishable from AC (Fig. 4C, Table 2). BFR/BS in $0.6 \times 30 \times 1$ mice was significantly greater than in $0.6 \times 15 \times 2$ mice. All other LIV groups within the $0.3 \mathrm{~g}$ and $0.6 \mathrm{~g}$ series were not significantly different from each other.

Mechanically induced greater BFR/BS in LIV groups when compared to controls was a result of either osteoblasts producing tissue faster, as indicated by increased MAR, or a consequence of more surfaces with osteoblasts, as indicated by an increase in MS/BS (Table 2). Not a single LIV group showed concomitant increases in MAR and MS/BS.

Bone Resorption (trabecular)

None of the six regimes using $0.3 \mathrm{~g}$ had a significant influence on trabecular Oc.S/BS (Table 2). Among the $0.6 \mathrm{~g}$ regimes, one daily bout of $15 \mathrm{~min}(0.6 \times 15 \times 1)$ caused $35 \%$ greater $(p<0.05)$ Oc.S/BS compared to AC without significant differences between individual LIV groups (Table 2).

Bone Quantity and Architecture (trabecular) 
Bone morphology in the trabecular metaphysis of mice exposed to $0.3 \mathrm{~g}$, one bout/d for $15 \mathrm{~min}$ $(0.3 \times 15 \times 1)$ was not different from age-matched controls (Table 2, Fig. 2). However, increasing bout number to two bouts per day while maintaining acceleration amplitude $(0.3 \times 15 \times 2)$ was associated with greater trabecular BV/TV (16\% over AC, $p<0.05$, Fig. 2 ) and greater Tb.N (9\% over AC, $p<0.05$, Table 2). Increasing the number of bouts per day to four yielded a trabecular structure that was comparable to controls. Similarly, one daily bout of $30 \mathrm{~min}(0.3 \times 30 \times 1)$ had no significant influence on bone morphology while increasing the number of daily bouts to two $(0.3 \times 30 \times 2)$ raised BV/TV by $12 \%$ over controls $(p<0.05$, Fig. 2$)$. At $0.6 \mathrm{~g}$, one LIV bout of $15 \mathrm{~min} / \mathrm{d}$ $(0.6 \times 15 \times 1)$ gave rise to $16 \%(p<0.0 .5)$ greater $B V / T V$ over controls. Experimental groups within the $0.3 \mathrm{~g}$ and 0.6 series were not significantly different from each other.

Bone Formation (cortical)

All mice displayed double labels at the endocortical surface of the metaphysis and the periosteal surface of the middiaphysis.

\section{$\underline{B F R / B S}$ in the cortical metaphysis}

At the endocortical surface of the metaphysis, one daily bout of $15 \mathrm{~min}$ at $0.3 \mathrm{~g}(0.3 \times 15 \times 1)$ had no significant influence on endocortical bone formation rates (Fig. 3A), while two bouts per day $(0.3 \times 15 \times 2)$ increased BFR/BS by $36 \%(p<0.05)$ over AC. Similar to trabecular bone, increasing bout number from two to four yielded BFR/BS level that were not different from those in AC. One daily $(0.3 \times 30 \times 1)$ and two daily bouts $(0.3 \times 30 \times 2)$ of 30 min increased BFR/BS by $36 \%(p<0.05)$ each. One daily vibration bout of $15 \mathrm{~min}$ at $0.6 \mathrm{~g}(0.6 \times 15 \times 1)$ increased BFR/BS by $34 \%$ compared to controls 
$(p<0.0 .5)$ (Fig. 3A). None of the other experimental groups displayed BFR/BS at the endocortical surface of the metaphysis that were either significantly different from controls or any given experimental group.

\section{$\underline{B F R / B S \text { in the middiaphysis }}$}

At the periosteal surface of the middiaphysis, exposure to $0.3 \mathrm{~g}$ vibrations for one, two, three, or four daily $15 \mathrm{~min}$ bouts failed to influence bone formation rates compared to AC (Fig. 3B). Similarly, $30 \mathrm{~min}$ or $60 \mathrm{~min}$ of single daily $0.3 \mathrm{~g}$ bouts were ineffective. In contrast, partitioning the single $0.3 \mathrm{~g} 60 \mathrm{~min}$ bout into two $(0.3 \times 30 \times 2)$ produced $73 \%$ greater $(\mathrm{p}<0.05) \mathrm{BFR} / \mathrm{BS}$ than in AC (Fig. 3B). For $0.6 \mathrm{~g}$ LIV signals, two bouts of $15 \mathrm{~min}$ each $(0.6 \times 15 \times 2)$ significantly increased BFR/BS over controls $(114 \%, \mathrm{p}<0.05)$ as well as over $0.6 \times 15 \times 1$ and $0.6 \times 30 \times 1$ regimes (Fig. 3 B).

\section{Cortical Bone Area and Geometry}

In the metaphysis, mice subjected to two bouts of $30 \mathrm{~min}$ LIV at $0.3 \mathrm{~g}(0.3 \times 30 \times 2)$ had a $7 \%$ greater $(p<0.05)$ periosteal area and $13 \%$ greater $(p<0.05)$ bone marrow area than controls (Table 5$)$. In $0.3 \times 30 \times 2$ mice, these two measures were also greater than in $0.3 \times 30 \times 1$ and $0.3 \times 60 \times 1$ mice. Two bouts of $15 \mathrm{~min}$ at $0.6 \mathrm{~g}(0.6 \times 15 \times 2)$ were associated with an $8 \%$ greater $(p<0.05)$ cortical bone area, a LIV response that was significantly different from $0.6 \times 30 \times 1$ mice (Table 5). In the middiaphysis, the only intervention that altered cortical bone properties with respect to controls were two bouts of $30 \mathrm{~min}$ at $0.3 \mathrm{~g}(0.3 \times 30 \times 2)$, resulting in $8 \%$ greater $(\mathrm{p}<0.05)$ total bone area (Table 5$)$. No significant differences between individual groups were detected. 


\section{Discussion}

We evaluated variables that may modulate the sensitivity of the growing musculoskeleton to LIV regimes and tested whether bone formation rates in the growing skeleton benefit from increasing the length of a bout, increasing the number of daily bouts of a given duration, partitioning one long daily bout into several discrete bouts, or doubling vibration magnitude. Three-way ANOVA across all LIV groups indicated that 30min and 60min LIV regimes produced trabecular bone formation rates in the proximal metaphysis of the tibia that were greater than for $15 \mathrm{~min}$ regimes. In contrast, the number of $\operatorname{LIV}$ bouts per day $(1,2$, or 4$)$ or acceleration magnitude $(0.3 \mathrm{~g}$ or $0.6 \mathrm{~g}$ ) was not a modulator of metaphyseal trabecular bone formation rates. In metaphyseal and diaphyseal cortical bone, differences between individual regimes were more subtle but the longest duration applied over a single session was ineffective for both acceleration magnitudes. Soleus muscle morphology, bone's chemical properties, and bone resorption were predominantly unaffected by any of the LIV treatments.

Trabecular bone formation rates of most LIV schemes were not significantly different between each other even though some signals induced significantly greater BFR/BS than seen in controls while other signal combinations did not. Overall integration of results from varying LIV magnitude, duration, and number of bouts demonstrated a pattern that is distinct from the doseresponse behavior commonly observed when skeletal tissues are exposed to low-frequency mechanical signals [31-33] extending results from previous in vivo [20,34] and in vitro [19, 35] investigations that did not find linear associations between vibration acceleration/frequency and the cellular response. Identification of the differences in mechanotransduction between low- 
and high-frequency mechanical signals may ultimately provide important clues towards a comprehensive optimization of LIV variables.

Because of the large number of variables defining a vibration regime [36], it is not practical to include all variables in a single study. For instance, the frequency of the signal was not varied here. This had the benefit of not introducing a potentially confounding variable through the intricate relation between signal frequency, bout duration, and cycle number but it needs to be considered that frequency (e.g., $45 \mathrm{~Hz}$ vs $90 \mathrm{~Hz}$ ) can have powerful effects on the efficacy of lowintensity vibrations in the musculoskeletal system $[21,23]$. We included two sham age-matched control groups but not six, the necessary number of groups to control for all combinations of number of bouts and bout durations. Therefore, we cannot exclude the possibility that a potential stress response to handling or interactions with other mice in the vibration container may have affected our results. Lastly, the 3wk experimental protocol was chosen as short enough to allow for effective quantification of changes in bone formation rates and long enough that changes in bone morphology could be detected if bone's response to the individual signals (and differences between them) was strongly anabolic. Our data suggest that, similar to previous LIV studies in the growing skeleton, protocol duration will need to be extended before greater structural changes can be observed $[6,7]$. Thus, changes in bone quantity and geometry/architecture were considered only a secondary outcome measure here.

Dynamic bone strain magnitudes increased from approximately $10 \mu \varepsilon$ to $30 \mu \varepsilon$ when LIV acceleration was raised from $0.3 \mathrm{~g}$ to $0.6 \mathrm{~g}$. While these data primarily provided an estimate of the relative difference in dynamic strain magnitudes as a function of acceleration magnitude and accurate tibial peak strains in a 8wk old mouse may be different [37], deformations were two 
orders of magnitude below levels that may induce damage in the bone matrix and at least one order of magnitude below previously proposed anabolic strain thresholds [38]. The lack of differences between the higher strain $0.6 \mathrm{~g}$ signals and the lower strain $0.3 \mathrm{~g}$ signals emphasizes results from in vitro $[18,35]$ and in vivo $[20,21]$ studies that suggested the mechanism by which bone senses LIV is not primarily dependent on bone strain magnitude.

Splitting a single mechanical loading session into discrete bouts that are distributed over the event of a day has been shown to enhance the efficacy of high-magnitude mechanical signals applied at low $(<10 \mathrm{~Hz})$ frequencies [39]. While cells subjected to LIV in culture may exhibit a similar phenomenon [17], we did not observe enhanced trabecular bone formation when bouts were partitioned. In contrast, a single session of $0.6 \mathrm{~g} \times 30 \mathrm{~min}$ provided greater trabecular bone formation than in controls but distributing this regime over two bouts engendered bone formations rates that were significantly smaller and similar to controls even though total duration and number of loading cycles were unchanged. The cause for these incongruent results may lie with a different mechanosensory mechanism by which low- and high-frequency mechanical signals are perceived in the skeleton and the different environment of cells in vitro versus in vivo. Using the same mouse model, we previously found that the inclusion of short rest periods (1015sec) after every 1 or 15 seconds of LIV renders the stimulus less effective [6, 7] even though incorporation of short rest periods has been a successful strategy for potentiating the cellular response to low-frequency mechanical regimes in vivo [40]. Together, these data suggest that the efficacy of LIV is dependent on the temporal continuity of a relatively large number of loading cycles with 30 -minute $(81,000$ cycles) and 60 -minute bouts $(162,000$ cycles) being more efficacious than $15 \mathrm{~min}$ bouts. 
Skeletal and muscular systems are interdependent and muscle-bone interactions have recently received considerable attention. Generally, morphologic adaptations of muscle to altered mechanical environments precede the adaptation of bone which often has been construed as evidence that bone responds to forces generated by muscle rather than those transferred as ground reaction forces [41]. Given that the mouse soleus can readily respond to 6wk of LIV treatment [7], the lack of changes in muscle histology in response to any of the $3 w k$ LIV regimes may suggest that muscle forces are not necessary for bone to sense LIV [36]. Regardless, both muscle and bone can respond to LIV [9] and understanding the mechanisms and perhaps interaction by which these two tissues may benefit from LIV will aid in the development of integrated musculoskeletal mechanical countermeasures.

The principal aim of this study was to determine LIV variables that may augment bone accretion during growth, the most opportune period to increase skeletal strength with exercise [42]. Inherently, results from this study may not apply to adult bone with largely quiescent surfaces. We found bout duration to be the most robust modulator of trabecular bone formation rates while number of daily bouts and acceleration magnitude had no significant influence across LIV signals considered here. Bone resorption and muscle morphology were largely unaffected by the LIV regimes and new bone formed during LIV retained its chemical quality. Our data may provide a basis for designing LIV regimes with greater efficacy but a potential non-linearity of the biologic response to LIV duration, number of daily bouts, and magnitude may need to be considered. 


\section{Acknowledgements}

Funding by the US Army Medical Research and Material Command (DAMD 17-03-1-0777), the Department of Defense (DoD W81XWH-14-1-0281), and the National Institutes of Health (NBIB EB01435101A) was greatly appreciated. 


\section{References}

1. Boreham CA, McKay HA (2011) Physical activity in childhood and bone health. British journal of sports medicine 45:877-879

2. Kelley GA, Kelley KS, Kohrt WM (2012) Effects of ground and joint reaction force exercise on lumbar spine and femoral neck bone mineral density in postmenopausal women: a metaanalysis of randomized controlled trials. BMC musculoskeletal disorders 13:177

3. Nordstrom P, Sievanen H, Gustafson Y, Pedersen NL, Nordstrom A (2013) High physical fitness in young adulthood reduces the risk of fractures later in life in men: A nationwide cohort study. J Bone Miner Res 28:1061-1067

4. Gunter KB, Almstedt HC, Janz KF (2012) Physical activity in childhood may be the key to optimizing lifespan skeletal health. Exerc Sport Sci Rev 40:13-21

5. Clark EM, Ness AR, Tobias JH (2008) Vigorous physical activity increases fracture risk in children irrespective of bone mass: a prospective study of the independent risk factors for fractures in healthy children. Journal of bone and mineral research 23:1012-1022

6. Xie L, Jacobson JM, Choi ES, Busa B, Donahue LR, Miller LM, Rubin CT, Judex S (2006) Lowlevel mechanical vibrations can influence bone resorption and bone formation in the growing skeleton. Bone 39:1059-1066

7. Xie L, Rubin C, Judex S (2008) Enhancement of the adolescent murine musculoskeletal system using low-level mechanical vibrations. J Appl Physiol 104:1056-1062

8. Vanleene M, Shefelbine SJ (2013) Therapeutic impact of low amplitude high frequency whole body vibrations on the osteogenesis imperfecta mouse bone. Bone 53:507-514

9. Gilsanz V, Wren TA, Sanchez M, Dorey F, Judex S, Rubin C (2006) Low-level, highfrequency mechanical signals enhance musculoskeletal development of young women with low BMD. J Bone Miner Res 21:1464-1474

10. Ligouri GC, Shoepe TC, Almstedt HC (2012) Whole Body Vibration Training is Osteogenic at the Spine in College-Age Men and Women. Journal of human kinetics 31:55-68

11. Lai C-L, Tseng S-Y, Chen C-N, Liao W-C, Wang C-H, Lee M-C, Hsu P-S (2013) Effect of 6 months of whole body vibration on lumbar spine bone density in postmenopausal women: a randomized controlled trial. Clinical interventions in aging 8:1603 
12. Ward K, Alsop C, Caulton J, Rubin C, Adams J, Mughal Z (2004) Low magnitude mechanical loading is osteogenic in children with disabling conditions. J Bone MinerRes 19:360-369

13. Reyes ML, Hernandez M, Holmgren L, Sanhueza E, Escobar RG (2011) High-frequency, low-intensity vibrations increase bone mass and muscle strength in upper limbs, improving autonomy in disabled children. J Bone Miner Res 26:1759-1766

14. Rubin C, Recker R, Cullen D, Ryaby J, McCabe J, McLeod K (2004) Prevention of postmenopausal bone loss by a low-magnitude, high-frequency mechanical stimuli: a clinical trial assessing compliance, efficacy, and safety. J Bone MinerRes 19:343-351

15. Brouwers JE, van Rietbergen B, Ito K, Huiskes R (2010) Effects of vibration treatment on tibial bone of ovariectomized rats analyzed by in vivo micro-CT. J Orthop Res 28:62-69

16. Slatkovska L, Alibhai SM, Beyene J, Hu H, Demaras A, Cheung AM (2011) Effect of 12 months of whole-body vibration therapy on bone density and structure in postmenopausal women: a randomized trial. Ann Intern Med 155:668-679, W205

17. Sen B, Xie Z, Case N, Styner M, Rubin CT, Rubin J (2011) Mechanical signal influence on mesenchymal stem cell fate is enhanced by incorporation of refractory periods into the loading regimen. Journal of Biomechanics 44:593-599

18. Uzer G, Manske SL, Chan ME, Chiang FP, Rubin CT, Frame MD, Judex S (2012) Separating Fluid Shear Stress from Acceleration during Vibrations in Vitro: Identification of Mechanical Signals Modulating the Cellular Response. Cellular and molecular bioengineering 5:266-276

19. Uzer G, Pongkitwitoon S, Ian C, Thompson WR, Rubin J, Chan ME, Judex S (2014) Gap Junctional Communication in Osteocytes Is Amplified by Low Intensity Vibrations In Vitro. PloS one 9:e90840

20. Christiansen BA, Silva MJ (2006) The effect of varying magnitudes of whole-body vibration on several skeletal sites in mice. Ann Biomed Eng 34:1149-1156

21. Judex S, Lei X, Han D, Rubin C (2007) Low-magnitude mechanical signals that stimulate bone formation in the ovariectomized rat are dependent on the applied frequency but not on the strain magnitude. J Biomech 40:1333-1339 
22. Pasqualini M, Lavet C, Elbadaoui M, Vanden-Bossche A, Laroche N, Gnyubkin V, Vico L (2013) Skeletal site-specific effects of whole body vibration in mature rats: from deleterious to beneficial frequency-dependent effects. Bone 55:69-77

23. Holguin N, Uzer G, Chiang FP, Rubin C, Judex S (2011) Brief daily exposure to low-intensity vibration mitigates the degradation of the intervertebral disc in a frequency-specific manner. J Appl Physiol 111:1846-1853

24. Robling AG, Hinant FM, Burr DB, Turner CH (2002) Improved bone structure and strength after long-term mechanical loading is greatest if loading is separated into short bouts. JBone MinerRes 17:1545-1554

25. DiPasquale DM, Cheng M, Billich W, Huang SA, van Rooijen N, Hornberger TA, Koh TJ (2007) Urokinase-type plasminogen activator and macrophages are required for skeletal muscle hypertrophy in mice. Am J Physiol Cell Physiol 293:C1278-1285

26. Fritton SP, McLeod KJ, Rubin CT (2000) Quantifying the strain history of bone: spatial uniformity and self- similarity of low-magnitude strains. JBiomech 33:317-325

27. Dempster DW, Compston JE, Drezner MK, Glorieux FH, Kanis JA, Malluche H, Meunier PJ, Ott SM, Recker RR, Parfitt AM (2013) Standardized nomenclature, symbols, and units for bone histomorphometry: a 2012 update of the report of the ASBMR Histomorphometry Nomenclature Committee. J Bone Miner Res 28:2-17

28. Liu C, Sanghvi R, Burnell JM, Howard GA (1987) Simultaneous demonstration of bone alkaline and acid phosphatase activities in plastic-embedded sections and differential inhibition of the activities. Histochemistry 86:559-565

29. Busa B, Miller LM, Rubin CT, Qin YX, Judex S (2005) Rapid establishment of chemical and mechanical properties during lamellar bone formation. Calcif Tissue Int 77:386-394

30. Bender R, Lange S (2001) Adjusting for multiple testing--when and how? J Clin Epidemiol 54:343-349

31. Rubin CT, Lanyon LE (1985) Regulation of bone mass by mechanical strain magnitude. CalcifTissue Int 37:411-417

32. Rubin CT, Lanyon LE (1984) Regulation of bone formation by applied dynamic loads. JBone Joint Surg[Am] 66:397-402 
33. Cullen DM, Smith RT, Akhter MP (2001) Bone-loading response varies with strain magnitude and cycle number. Journal of applied physiology 91:1971-1976

34. Garman R, Gaudette G, Donahue LR, Rubin C, Judex S (2007) Low-level accelerations applied in the absence of weight bearing can enhance trabecular bone formation. J Orthop Res 25:732-740

35. Uzer G, Pongkitwitoon S, Ete Chan M, Judex S (2013) Vibration induced osteogenic commitment of mesenchymal stem cells is enhanced by cytoskeletal remodeling but not fluid shear. J Biomech 46:2296-2302

36. Judex S, Rubin CT (2010) Is bone formation induced by high-frequency mechanical signals modulated by muscle activity? J Musculoskelet Neuronal Interact 10:3-11

37. Gross TS, McLeod KJ, Rubin CT (1992) Characterizing bone strain distributions in vivo using three triple rosette strain gages. JBiomech 25:1081-1087

38. Frost HM (1987) Bone "mass" and the "mechanostat": a proposal. Anatomical Record 219:1-9

39. Robling AG, Burr DB, Turner CH (2000) Partitioning a daily mechanical stimulus into discrete loading bouts improves the osteogenic response to loading. Journal of Bone and Mineral Research 15:1596-1602

40. Gross TS, Poliachik SL, Ausk BJ, Sanford DA, Becker BA, Srinivasan S (2004) Why rest stimulates bone formation: a hypothesis based on complex adaptive phenomenon. ExercSport SciRev 32:9-13

41. Robling AG (2009) Is bone's response to mechanical signals dominated by muscle forces? MedSciSports Exerc 41:2044-2049

42. Rizzoli R, Bianchi ML, Garabédian M, McKay HA, Moreno LA (2010) Maximizing bone mineral mass gain during growth for the prevention of fractures in the adolescents and the elderly. Bone 46:294-305 
Tables

Table 1. Overview of experimental groups and daily exposure schedule.

\begin{tabular}{|c|c|c|c|c|c|c|c|c|c|c|c|}
\hline \multicolumn{2}{|r|}{ Group } & 9am & 10am & $11 \mathrm{am}$ & $12 \mathrm{pm}$ & $1 \mathrm{pm}$ & $2 \mathrm{pm}$ & $3 p m$ & $4 p m$ & $5 \mathrm{pm}$ & $6 p m$ \\
\hline \multirow{2}{*}{$\begin{array}{l}\text { Og } \\
A C\end{array}$} & $1 \times 15 \min (n=12)$ & & & & & & & & & & \\
\hline & $2 \times 30 \min (n=12)$ & & & & & & & & & & \\
\hline \multirow{6}{*}{$0.3 \mathrm{~g}$} & $1 \times 15 \min (n=11)$ & & & & & & & & & & \\
\hline & $2 \times 15 \min (n=11)$ & & & & & & & & & & \\
\hline & $4 \times 15 \min (n=12)$ & & & & & & & & & & \\
\hline & $1 \times 30 \min (n=12)$ & & & & & & & & & & \\
\hline & $2 \times 30 \min (n=11)$ & & & & & & & & & & \\
\hline & $1 \times 60 \min (n=12)$ & & & & & & & & & & \\
\hline \multirow{3}{*}{$0.6 \mathrm{~g}$} & $1 \times 15 \min (n=11)$ & & & & & & & & & & \\
\hline & $2 \times 15 \min (n=11)$ & & & & & & & & & & \\
\hline & $1 \times 30 \min (n=11)$ & & & & & & & & & & \\
\hline
\end{tabular}

The width of each black bar is proportional to the loading duration. The two sham age-matched-control groups $(A C)$ were exposed to an inactive $(\mathrm{Og})$ plate. Sham treatments are presented by grey bars. 
Table 2. Body mass and trabecular histomorphometric/architectural endpoints (mean $\pm S D$ ).

\begin{tabular}{|c|c|c|c|c|c|c|c|c|c|}
\hline & $\begin{array}{c}\text { Body } \\
\text { Mass (g) }\end{array}$ & $\begin{array}{l}\text { Length } \\
(\mathrm{mm})\end{array}$ & $\begin{array}{c}\text { Oc.S/BS } \\
(\%)\end{array}$ & $\begin{array}{c}\text { MAR } \\
(\mu \mathrm{m} / d)\end{array}$ & $\begin{array}{c}M S / B S \\
(\%)\end{array}$ & $\begin{array}{c}B F R / B S \\
\left(\mu m^{3} /\right. \\
\left.\mu m^{2} / y r\right)\end{array}$ & $\begin{array}{c}\text { Conn.D } \\
\left(1 / \mathrm{mm}^{3}\right)\end{array}$ & $\begin{array}{c}T b . N \\
(1 / \mathrm{mm})\end{array}$ & $\begin{array}{l}\text { Tb.Th } \\
(\mu m)\end{array}$ \\
\hline$B C$ & $23.2 \pm 0.9 *$ & $15.9 \pm 0.6^{*}$ & $16.6 \pm 3.8$ & $1.8 \pm 0.1^{*}$ & $17.6 \pm 3.0^{*}$ & $114 \pm 24 *$ & $309 \pm 54$ & $6.4 \pm 0.3$ & $37.1 \pm 1.1^{*}$ \\
\hline$A C$ & $27.5 \pm 1.2$ & $16.8 \pm 0.5$ & $17.1 \pm 3.9$ & $1.1 \pm 0.2$ & $12.4 \pm 5.4$ & $49 \pm 19$ & $301 \pm 65$ & $6.3 \pm 0.5$ & $43.2 \pm 2.1$ \\
\hline $0.3 \times 15 \times 1$ & $27.7 \pm 1.3$ & $16.6 \pm 0.5$ & $20.4 \pm 4.0$ & $1.5 \pm 0.3^{*}$ & $11.6 \pm 4.0$ & $63 \pm 23$ & $311 \pm 65$ & $6.3 \pm 0.5$ & $44.4 \pm 1.1$ \\
\hline $0.3 \times 15 \times 2$ & $27.5 \pm 0.8$ & $16.8 \pm 0.4$ & $20.1 \pm 6.6$ & $1.6 \pm 0.3^{*}$ & $13.4 \pm 3.0$ & $79 \pm 21 *$ & $370 \pm 70$ & $6.8 \pm 0.3^{*}$ & $43.3 \pm 3.0$ \\
\hline $0.3 \times 15 \times 4$ & $26.7 \pm 1.2$ & $16.9 \pm 0.3$ & $19.8 \pm 6.0$ & $1.1 \pm 0.2^{\dagger}$ & $15.7 \pm 3.6$ & $63 \pm 21$ & $342 \pm 79$ & $6.5 \pm 0.6$ & $43.6 \pm 1.3$ \\
\hline $0.3 \times 30 \times 1$ & $26.6 \pm 1.3$ & $16.6 \pm 0.5$ & $16.9 \pm 7.0$ & $1.3 \pm 0.3^{\dagger}$ & $18.9 \pm 4.9 *^{\dagger}$ & $90 \pm 38 *$ & $291 \pm 68$ & $6.1 \pm 0.5$ & $43.8 \pm 3.5$ \\
\hline $0.3 \times 30 \times 2$ & $28.4 \pm 1.4$ & $17.1 \pm 0.3$ & $19.9 \pm 6.0$ & $1.2 \pm 0.2^{\dagger}$ & $18.8 \pm 5.6^{* \dagger}$ & $90 \pm 34 *$ & $359 \pm 21$ & $6.6 \pm 0.3^{* \#}$ & $44.0 \pm 2.5$ \\
\hline $0.3 \times 60 \times 1$ & $26.5 \pm 1.8$ & $16.5 \pm 0.6$ & $20.8 \pm 6.0$ & $1.2 \pm 0.3^{\dagger}$ & $20.7 \pm 6.6^{*}$ & $92 \pm 38^{*}$ & $306 \pm 28$ & $6.3 \pm 0.3^{-}$ & $44.1 \pm 1.9$ \\
\hline $0.6 \times 15 \times 1$ & $27.6 \pm 1.7$ & $16.7 \pm 0.3$ & $23.1 \pm 6.1 *$ & $1.6 \pm 0.4^{*+}$ & $13.3 \pm 2.5^{+}$ & $77 \pm 21^{*+}$ & $343 \pm 40$ & $6.6 \pm 0.3$ & $44.3 \pm 1.7$ \\
\hline $0.6 \times 15 \times 2$ & $28.0 \pm 1.3$ & $16.6 \pm 0.5$ & $17.3 \pm 6.5$ & $1.6 \pm 0.4^{*+}$ & $11.2 \pm 2.4^{+}$ & $63 \pm 16^{+}$ & $336 \pm 61$ & $6.3 \pm 0.4$ & $43.8 \pm 2.7$ \\
\hline $0.6 \times 30 \times 1$ & $27.1 \pm 1.1$ & $16.8 \pm 0.3$ & $17.9 \pm 4.5$ & $1.2 \pm 0.1$ & $21.2 \pm 3.9 *$ & $96 \pm 21 *$ & $318 \pm 61$ & $6.4 \pm 0.5$ & $44.3 \pm 2.1$ \\
\hline $\begin{array}{l}\text { *: Differe } \\
{ }^{+} \text {: Differer } \\
{ }^{+} \text {: Differer } \\
{ }^{-} \text {: Differen } \\
{ }^{*} \text { : Differer }\end{array}$ & $\begin{array}{l}\text { from } A C \\
\text { from } 0.6 x \\
\text { from } 0.3 x \\
\text { from } 0.3 x\end{array}$ & $\begin{array}{l}30 \times 1 \\
15 \times 1 \text { and } 0 \\
5 \times 2 \\
30 \times 1\end{array}$ & $3 \times 15 \times 2$ & & & & & & \\
\hline
\end{tabular}


Table 3. Chemical properties (mean $\pm S D$ ) of newly mineralized bone in trabecular and cortical bone of the tibial metaphysis in age-matched control and a vibrated group that showed significant increases in bone formation rates $(0.3 \times 15 \times 2)$.

\begin{tabular}{lcccc} 
& \multicolumn{2}{c}{ Trabecular } & \multicolumn{2}{c}{ Cortical } \\
\hline & $A C$ & $0.3 \times 15 \times 2$ & $A C$ & $0.3 \times 15 \times 2$ \\
& $\left(10^{-3}\right)$ & $\left(10^{-3}\right)$ & $\left(10^{-3}\right)$ & $\left(10^{-3}\right)$ \\
\hline Phosphate/Protein & $540 \pm 185$ & $520 \pm 110$ & $626 \pm 181$ & $638 \pm 215$ \\
Carbonate·Protein & $66 \pm 12$ & $68 \pm 7$ & $70 \pm 18$ & $68 \pm 15$ \\
Carbonate-Phosphate & $136 \pm 49$ & $141 \pm 27$ & $116 \pm 47$ & $114 \pm 29$ \\
Acid Phosphate-Total Phosphate & $3.7 \pm 0.9$ & $3.7 \pm 0.8$ & $3.9 \pm 0.8$ & $3.7 \pm 1.0$ \\
Crystallinity & $985 \pm 76$ & $1044 \pm 105$ & $815 \pm 100$ & $818 \pm 129$ \\
Collagen Cross-Linking ratio & $1548 \pm 184$ & $1604 \pm 158$ & $1655 \pm 128$ & $1705 \pm 172$
\end{tabular}


Table 4. Muscle histologic properties in the soleus (mean $\pm S D$ ).

\begin{tabular}{cccccc} 
& $\begin{array}{c}\text { Total } \\
\text { area }\left(\mathrm{mm}^{2}\right)\end{array}$ & $\begin{array}{c}\text { Total fiber } \\
\text { number }\end{array}$ & $\begin{array}{c}\text { Type I } \\
\text { number }\end{array}$ & $\begin{array}{c}\text { Type II } \\
\text { number }\end{array}$ & $\begin{array}{c}\text { Type-I/Type-II } \\
(\%)\end{array}$ \\
\hline BC & $0.99 \pm 0.18$ & $627 \pm 102$ & $165 \pm 35$ & $441 \pm 70$ & $35.8 \pm 3.8$ \\
AC & $0.99 \pm 0.22$ & $641 \pm 125$ & $171 \pm 37$ & $470 \pm 101$ & $36.9 \pm 7.7$ \\
\hline $0.3 \times 15 \times 1$ & $0.91 \pm 0.23$ & $616 \pm 160$ & $157 \pm 39$ & $457 \pm 116$ & $36.5 \pm 7.0$ \\
$0.3 \times 15 \times 2$ & $0.96 \pm 0.23$ & $690 \pm 97$ & $182 \pm 30$ & $508 \pm 80$ & $36.3 \pm 5.6$ \\
$0.3 \times 15 \times 4$ & $1.15 \pm 0.27$ & $700 \pm 121$ & $181 \pm 24$ & $518 \pm 100$ & $35.6 \pm 4.4$ \\
$0.3 \times 30 \times 1$ & $1.12 \pm 0.26$ & $682 \pm 62$ & $190 \pm 36$ & $492 \pm 53$ & $39.1 \pm 8.6$ \\
$0.3 \times 30 \times 2$ & $1.01 \pm 0.23$ & $683 \pm 100$ & $174 \pm 28$ & $508 \pm 83$ & $34.8 \pm 6.0$ \\
$0.3 \times 60 \times 1$ & $0.98 \pm 0.20$ & $685 \pm 117$ & $181 \pm 32$ & $507 \pm 93$ & $36.8 \pm 6.1$ \\
$0.6 \times 15 \times 1$ & $0.96 \pm 0.23$ & $630 \pm 160$ & $172 \pm 52$ & $458 \pm 114$ & $37.3 \pm 7.3$ \\
$0.6 \times 15 \times 2$ & $0.88 \pm 0.19$ & $638 \pm 74$ & $165 \pm 30$ & $473 \pm 65$ & $35.4 \pm 7.6$ \\
$0.6 \times 30 \times 1$ & $0.96 \pm 0.13$ & $711 \pm 85$ & $181 \pm 34$ & $520 \pm 71$ & $37.2 \pm 4.5$
\end{tabular}


Table 5. Cortical bone properties of the metaphysis and diaphysis (mean $\pm S D$ ).

\begin{tabular}{|c|c|c|c|c|c|c|}
\hline & \multicolumn{3}{|c|}{ Metaphysis } & \multicolumn{3}{|c|}{ Diaphysis } \\
\hline & $\begin{array}{c}\text { Tt.Ar } \\
\left(\mathrm{mm}^{2}\right)\end{array}$ & $\begin{array}{c}\text { Ct.Ar } \\
\left(\mathrm{mm}^{2}\right)\end{array}$ & $\begin{array}{l}\text { Ma.Ar } \\
\left(\mathrm{mm}^{2}\right)\end{array}$ & $\begin{array}{c}\text { Tt.Ar } \\
\left(\mathrm{mm}^{2}\right)\end{array}$ & $\begin{array}{c}\text { Ct.Ar } \\
\left(\mathrm{mm}^{2}\right)\end{array}$ & $\begin{array}{l}\text { Ma.Ar } \\
\left(\mathrm{mm}^{2}\right)\end{array}$ \\
\hline$B C$ & $3.36 \pm 0.35$ & $1.05 \pm 0.05$ & $2.31 \pm 0.30$ & $0.84 \pm 0.08$ & $0.56 \pm 0.04^{*}$ & $0.28 \pm 0.04$ \\
\hline$A C$ & $3.28 \pm 0.28$ & $1.07 \pm 0.11$ & $2.21 \pm 0.26$ & $0.88 \pm 0.05$ & $0.63 \pm 0.03$ & $0.26 \pm 0.03$ \\
\hline $0.3 \times 15 \times 1$ & $3.40 \pm 0.18$ & $1.08 \pm 0.07$ & $2.31 \pm 0.17$ & $0.88 \pm 0.06$ & $0.62 \pm 0.03$ & $0.26 \pm 0.03$ \\
\hline $0.3 \times 15 \times 2$ & $3.49 \pm 0.18$ & $1.14 \pm 0.08$ & $2.35 \pm 0.17$ & $0.88 \pm 0.07$ & $0.62 \pm 0.04$ & $0.27 \pm 0.03$ \\
\hline $0.3 \times 15 \times 4$ & $3.36 \pm 0.20$ & $1.04 \pm 0.08$ & $2.32 \pm 0.19$ & $0.92 \pm 0.06$ & $0.64 \pm 0.04$ & $0.28 \pm 0.03$ \\
\hline $0.3 \times 30 \times 1$ & $3.20 \pm 0.24^{+}$ & $1.00 \pm 0.12$ & $2.20 \pm 0.16^{+}$ & $0.89 \pm 0.07$ & $0.63 \pm 0.04$ & $0.26 \pm 0.04$ \\
\hline $0.3 \times 30 \times 2$ & $3.51 \pm 0.25^{*}$ & $1.02 \pm 0.14$ & $2.49 \pm 0.13^{*}$ & $0.95 \pm 0.06 *$ & $0.67 \pm 0.04$ & $0.29 \pm 0.02$ \\
\hline $0.3 \times 60 \times 1$ & $3.23 \pm 0.24^{+}$ & $1.03 \pm 0.07$ & $2.20 \pm 0.22^{+}$ & $0.89 \pm 0.06$ & $0.62 \pm 0.03$ & $0.27 \pm 0.04$ \\
\hline $0.6 \times 15 \times 1$ & $3.40 \pm 0.27$ & $1.12 \pm 0.12$ & $2.28 \pm 0.16$ & $0.88 \pm 0.07$ & $0.63 \pm 0.04$ & $0.26 \pm 0.03$ \\
\hline $0.6 \times 15 \times 2$ & $3.46 \pm 0.18$ & $1.15 \pm 0.07^{*}$ & $2.31 \pm 0.16$ & $0.92 \pm 0.07$ & $0.65 \pm 0.04$ & $0.26 \pm 0.03$ \\
\hline $0.6 \times 30 \times 1$ & $3.36 \pm 0.19$ & $1.02 \pm 0.06^{\dagger}$ & $2.34 \pm 0.16$ & $0.90 \pm 0.05$ & $0.61 \pm 0.04$ & $0.28 \pm 0.02$ \\
\hline *: Different & $\begin{array}{l}\text { rom } A C \\
\text { rom } 0.3 \times 30 \times 2 \\
\text { rom } 0.6 \times 15 \times 2\end{array}$ & & & & & \\
\hline
\end{tabular}




\section{Figures}

Figure 1. (A) Accelerometer recording of the vertically oscillating vibrating plate producing peak accelerations of $0.3 \mathrm{~g}$ or $0.6 \mathrm{~g}$ at $45 \mathrm{~Hz}$. (B) Simultaneous recording from a longitudinal strain gage attached to the antero-medial surface of the tibia while the mouse was subjected to $0.3 \mathrm{~g}$ and $0.6 \mathrm{~g}$ LIV or sitting on an inactive plate $(0 \mathrm{~g})$. A compressive strain of approximately $-70 \mu \varepsilon$ was produced by the weight of the mice $(0 \mathrm{~g})$. While the $0.3 \mathrm{~g}$ signal recorded from the accelerometer was not perfectly sinusoidal, it was sufficiently robust to generate a sinuoidal signal from the strain gage.

Figure 2. Trabecular bone volume fraction of the proximal tibial metaphysis in control and LIV groups (mean+SD). The dashed line represents the value of the baseline control group (BC, mean \pm SD). *: different from AC.

Figure 3. Bone formation rates in control and experimental groups (mean+SD) measured at the (A) endocortical surface of the proximal metaphasis and (B) periosteal surface of the middiaphysis. The dashed line represents the value of the baseline control group (BC, mean \pm SD). *: different from AC. ${ }^{\#}$ : different from $0.6 \times 15 \times 2$.

Figure 4. Relative differences in metaphyseal BFR/BS between LIV groups and age-matched controls (mean+SD). Presented are data from the nine LIV groups stratified to illustrate the effect of (A) duration per bout, (B) number of daily bouts, and (C) splitting a given daily treatment duration ( $30 \mathrm{~min}$ or $60 \mathrm{~min}$ ) into discrete multiple bouts. Individual groups may appear multiple times across the three figures. The age-matched control group is identical in figures A-C. * : different from AC. ${ }^{\wedge}$ : different from $0.6 \times 15 \times 2$. 